\title{
Raffaele de Cesare, Balzac e la lingua italiana, in «L'Analisi linguistica e letteraria»
}

\section{Marco Stupazzoni}

\section{Q OpenEdition}

1 Journals

\section{Edizione digitale}

URL: http://journals.openedition.org/studifrancesi/7632

DOI: 10.4000/studifrancesi.7632

ISSN: 2421-5856

\section{Editore}

Rosenberg \& Sellier

\section{Edizione cartacea}

Data di pubblicazione: 1 décembre 2009

Paginazione: 648

ISSN: 0039-2944

\section{Notizia bibliografica digitale}

Marco Stupazzoni, «Raffaele de Cesare, Balzac e la lingua italiana, in «L'Analisi linguistica e letteraria»», Studi Francesi [Online], 159 (LIII | III) | 2009, online dal 30 novembre 2015, consultato il 08 janvier 2021 URL: http://journals.openedition.org/studifrancesi/7632; DOI: https://doi.org/10.4000/studifrancesi. 7632

Questo documento è stato generato automaticamente il 8 janvier 2021.

\section{(c)}

Studi Francesi è distribuita con Licenza Creative Commons Attribuzione - Non commerciale - Non opere derivate 4.0 Internazionale. 


\title{
Raffaele de Cesare, Balzac e la lingua italiana, in «L'Analisi linguistica e letteraria»
}

\author{
Marco Stupazzoni
}

\section{NOTIZIA}

RAFFAELE DE CESARE, Balzac e la lingua italiana, in «L'Analisi linguistica e letteraria», anno XV, n², 2007 [ma pubblicato nel novembre 2008], pp. 272-309.

1 Nessuno meglio di Raffaele de Cesare avrebbe potuto affrontare e portare a miglior compimento questa ricerca su un tema così complesso e così poco esplorato dalla critica come quello relativo alla presenza e alla diffusione della lingua italiana nell'intera opera di Balzac. A dire il vero, in anni recenti, già M. T.Zanola, in un meritorio lavoro pubblicato nel 2005, aveva trattato con chiarezza di questa delicata questione - limitatamente però alla sola Comédie humaine - offrendo per la prima volta in maniera precisa valide e promettenti indicazioni circa l'indole "italianisante" di Balzac romanziere.

Maggiormente ampia e profonda è la prospettiva di analisi assunta da de Cesare per mettere in luce, in questo studio, «il livello delle conoscenze che Balzac aveva della lingua italiana, la quantità e la qualità delle sue citazioni transalpine, il compiacimento più o meno giustificato (o, per meglio dire, il vezzo accarezzato) che egli aveva di porle in mostra sotto l'occhio dei suoi lettori» (p. 273). Privo di qualsiasi base lessicografica, grammaticale e sintattica in lingua italiana che potesse derivare dalla sua formazione scolastica, Balzac ebbe modo, grazie soprattutto alle relazioni con un nutrito gruppo di amici italiani o "italianisants" e alle diverse suggestioni ed intermediazioni italofrancesi di provenienza musicale (Cimarosa, Zingarelli, Donizetti, Bellini, Rossini), di cogliere e di trasmettere alcuni echi della nostra lingua. In questo gruppo di amici e conoscenti, un posto di primo piano spetta a Latouche (l'autore di Fragoletta) senza 
dimenticare i nomi di personalità altrettanto importanti e determinanti quali il barone Gérard, il conte milanese Emilio Guidoboni-Visconti, il duca Edouard de Fitz-James. Altre figure da ricordare sono il duca Napoléon d'Abrantès, oltre alle amiche Zulma Carraud, Laure d'Abrantès e Fanny Sanseverino-Vimercati.

3 Passando poi ad allestire, secondo un ordine che, pur nel rispetto della scansione cronologica delle opere, assume come fondamento quello del genere della produzione balzachiana (di invenzione narrativa, di critica letteraria o politica, di comunicazione epistolare), l'autore motiva questa sua scelta metodologica ritenendo che l'italiano di Balzac «non ha [...] uno sviluppo che cammini di pari passo con gli anni, che si arricchisca, cioè, grazie alle sue successive esperienze di lettura e di vita vissuta nelle vicende personali acquisite nel corso dei suoi numerosi viaggi in Italia» (p. 277).

Nella prima sezione di questo suggestivo repertorio, vengono analizzati non solo $i$ cosiddetti 'romans de jeunesse' ma anche quei racconti o frammenti narrativi redatti dallo scrittore dalla seconda metà del 1830 alla fine del 1831 e rimasti inediti o pubblicati in riviste. Se le citazioni in lingua italiana tratte da questi scritti di apprendistato letterario non sembrano particolarmente frequenti e significative, più nutrito è l'insieme dei riferimenti all'italiano presenti nelle prime Scènes de la vie privée (Le Bal de Sceaux, La Vendetta, Sarrasine), nella Peau de chagrin, ne Les Marana e nella trilogia Histoire des Treize, mentre degno di nota è il caso di Père Goriot in cui Balzac attribuisce a Vautrin una solida conoscenza della nostra lingua. Particolarmente ricchi di riferimenti all'italiano sono i romanzi e i racconti redatti tra il 1836 e il 1838, negli anni, cioè, in cui il romanziere francese iniziò e approfondì le sue esperienze di viaggio nelle città della nostra Penisola, mentre per quel che riguarda la produzione narrativa successiva occorre sottolineare il fatto che, pur essendo in queste opere presenti citazioni e locuzioni italiane, "esse danno l'esempio di tediose repliche di parole e di modi di agire già largamente sfruttati» (p. 296). Un caso particolare ed abbastanza singolare è costituito dal romanzo Albert Savarus (1842) dove, osserva de Cesare, «la nostra lingua si riserva una larga porzione dell'invenzione narrativa» (p. 298). Complessivamente sommaria e priva di marcata originalità è la messe di citazioni presente nei romanzi della Comédie humaine posteriori al 1845 e negli scritti raccolti nelle Oeuvres diverses; più rilevante ed interessante è invece il repertorio di parole italiane desumibili dalla Correspondance in cui «appellativi di cortesia, attestazioni di calore umano, di amicizia, di affetto sgorgano in un vero e proprio effluvio» (p. 305).

5 Se, dunque, l'insieme delle citazioni in lingua italiana presenti nell'opera omnia balzachiana raggiunge all'incirca le mille e cinquecento occorrenze, non bisogna per questo esagerare, osserva bene de Cesare, «il valore che assume l'imponenza di tale quantità» (p.308). La loro qualità, infatti, si rivela assai tenue e di scarso pregio: numerosi sono i termini che, pur appartenendo «ad un linguaggio comune, colloquiale, quotidiano» o provenendo da diversi canali culturali restano comunque «incapaci di illuminare di una luce inattesa, folgorante la parola prescelta imprimendole quella incisività che la sua corrispondente francese non avrebbe saputo garantirle» (p. 309). In altri termini, conclude de Cesare, «Balzac appare - dispiaccia o non - più proclive a far mostra delle sue cognizioni linguistiche italiane che non a dominarle con esperta sicurezza» (ibid.). 